\title{
HIV and Sexual Health Services Available to Sexual and Gender Minority Youth Seeking Care at Outpatient Public Mental Health Programs in Two California Counties
}

\author{
Donald Clermont, ${ }^{1}$ Todd Gilmer, ${ }^{1}$ Jose Luis Burgos, ${ }^{1}$ Emily Berliant, ${ }^{1}$ and Victoria D. Ojeda ${ }^{1,2, *}$
}

\begin{abstract}
Purpose: Sexual and gender minority youth (SGMY, ages 16-24 years) face disparities in sexually transmitted infections (STIS) and HIV, in part, due to exposure to settings and behaviors that may harm youth's physical and mental health. This study examines the scope of sexual health and HIV services available to youth living with serious mental illness (SMI), including SGMY, seeking care at publicly funded outpatient mental health programs.

Methods: Between 2018 and 2019, we surveyed 183 managers of mental health programs serving youth living with SMI of ages 16-24 years, including SGMY, in San Diego and Los Angeles counties. Participants reported on programs' target populations, sexual health/HIV service provision, and the use of peer providers. Descriptive statistics and Pearson chi-square tests were used to describe sexual health/HIV services and identify programmatic characteristics associated with providing these services.

Results: Overall, 46\% of all programs surveyed provided sexual health/HIV services. Of these, $62 \%$ provided HIV education, $81 \%$ provided sexual/reproductive health education, and 69\% provided sexual/reproductive health education tailored for lesbian, gay, bisexual, queer, intersex ( $L G B Q I)$ youth. Peers often provided these services Chi-squared tests showed that programs employing peer specialists $(p=0.009)$ and targeting LGBQI youth ( $p=0.045$ ) were significantly more likely to provide sexual health/HIV services.

Conclusion: The use of peer providers may reduce stigma around sexual/HIV service utilization and promote SGMY's trust. Publicly funded outpatient mental health programs serving youth and especially those actively engaging SGMY may consider also offering onsite HIV, STI, and sexual health services, creating a one-stop-shop approach.
\end{abstract}

Keywords: sexual and gender minority; public mental health; HIV; youth; young adult

\section{Introduction}

Sexual and gender minority youth (SGMY; e.g., lesbian, gay, bisexual, transgender, queer, intersex, of ages 16-24 years) experience overlapping social identities (e.g., gender, sexual orientation, race/ethnicity, and immigrant status) due to the diverse social groups to which they belong. ${ }^{1-4}$ However, exclusion, stigmatization, and discrimination of these intersecting identities may ad- versely impact SGMY's mental and physical health outcomes. ${ }^{1,5}$ Specifically, SGMY are at high risk of experiencing mental health disparities while also being at disproportionate risk for sexually transmitted infections (STIs) and HIV. ${ }^{6-9}$ This study describes the availability of sexual health, STI, and HIV services by publicly funded mental health programs that serve SGMY in Southern California, a highly diverse region.

\footnotetext{
Departments of ${ }^{1}$ Family Medicine and Public Health and ${ }^{2}$ Medicine, University of California, San Diego School of Medicine, La Jolla, California, USA.
}

*Address correspondence to: Victoria D. Ojeda, PhD, MPH, Department of Family Medicine and Public Health, San Diego School of Medicine, 9500 Gilman Drive, La Jolla, CA 92037-0725, USA, E-mail: vojeda@health.ucsd.edu

(c) Donald Clermont et al., 2020; Published by Mary Ann Liebert, Inc. This Open Access article is distributed under the terms of the Creative Commons License (http://creativecommons.org/licenses/by/4.0), which permits unrestricted use, distribution, and reproduction in any medium, provided the original work is properly cited. 
SGMY are significantly more likely to experience conditions that can elevate the likelihood of mental and physical health disparities. Stigma and discrimination by family, peers, and community members due to youth's gender and sexual orientation contribute to adverse mental health outcomes among SGMY, ${ }^{10-13}$ including higher rates of suicidality, depression, and anxiety disorders. ${ }^{12,14-16}$ SGMY experience higher rates of STIs and HIV than heterosexual/cisgender youth, ${ }^{6-9}$ in part because of engagement in risky sexual behaviors (e.g., infrequent condom use, multiple sexual partners, and trading sex) and higher rates of alcohol and illicit drug use-potentially as coping mechanism, which may subsequently contribute to unsafe sexual practices. ${ }^{17-24}$ Importantly, systematic discrimination, such as bullying and physical and sexual abuse, including forced sex or sexual dating violence, has resulted in the overrepresentation of SGMY among homeless or housing insecure youth. ${ }^{6,25-32}$ Notably, African American and Latino SGMY are disproportionately represented among those seeking homelessness services. ${ }^{26,33}$ The numerous adverse social conditions encountered by SGMY can exacerbate their health burden and underscore their need for comprehensive services that can safeguard their mental and physical health.

SGMY experience numerous barriers to receiving needed physical and mental health services (HSs). The Gelberg-Andersen Behavioral Model of Health Services Utilization for Vulnerable Populations can elucidate the factors underlying HS use disparities among SGMY. ${ }^{34,35}$ The Gelberg-Andersen model has been used to study HS utilization among vulnerable communities such as adults experiencing homelessness or serious mental illness (SMI) ${ }^{36-38}$ The model notes that predisposing (e.g., demographics), enabling (e.g., individual and community resources), and need characteristics (e.g., individual perceived need and professionally evaluated need) interact to influence health care utilization. Enabling factors play an important role in youth's health care access as they may experience disruptions in health care as they exit child/ adolescent HSs and enter into adult HSs. The model further identifies areas of vulnerability relevant to SGMY such as sexual orientation, victimization, family resources, and youth's access to public benefits. ${ }^{39,40}$ Access to care barriers for youth includes confusion about navigating the insurance system or the cost of coverage, thus financial barriers may be important factors limiting youth's HS utilization. ${ }^{41,42}$ Characteristics of the health system may also impact SGMY's engagement and range from limited provider training regarding SGM health to stigmatization of SGM individuals and assumptions of patients' heteronormativity by clinicians. ${ }^{42}$ Consequently, SGMY may mistrust their primary care provider and fear revealing their sexual orientation, resulting in less disclosure about their sexual health needs, HIV risk behaviors, or other factors that elevate SGMY's vulnerability to poor health outcomes. Collectively, this array of individual, family, community, and health system-level factors may contribute to SGMY's unmet needs across physical and social domains that influence health (e.g., conflict management/ reduction within families). ${ }^{42-45}$

California has made important progress in the provision of youth-focused mental HSs, facilitated, in part, by the 2004 Mental Health Services Act. ${ }^{46,47}$ For example, in 2017, 30.8\% of California's community mental health centers offered treatment programs or groups designed exclusively for youth and SGMY (19.7\%) versus $17.8 \%$ and $15.7 \%$, respectively, of community mental health centers throughout the United States. ${ }^{48}$ HIV services that are offered by mental health programs enhance care and reduce barriers to accessing needed sexual HSs. ${ }^{49}$ Moreover, integrating sexual health, including HIV services, into mental health care may improve both mental and sexual health outcomes among SGMY by reducing social, administrative, logistical, and financial barriers to care. ${ }^{49,50}$ Yet, we continue to lack a nuanced understanding of the role that mental health programs play in providing sexual health, STI, and HIV services to SGMY who seek mental health care.

Peer-led services have the potential to support SGMY's access to and use of sexual and mental HSs. HIV programs have consistently engaged peers to provide sex education community outreach, and HIV testing. ${ }^{51-56}$ SGMY consider peer support (PS) and guidance to be critical aspects of sexual HSs. ${ }^{56-59}$ and PS may improve health and social outcomes by raising self-efficacy and self-confidence. ${ }^{60,61}$ In addition, those receiving PS exhibit a higher likelihood of returning for annual visits, reductions in risky sexual behavior, and increased self-reported empowerment. ${ }^{59-61}$ PS is increasingly used within mental health settings as they are considered to provide culturally and developmentally appropriate support systems that build on mutuality, empathy, and trust. ${ }^{62-64}$ In $2017,32.0 \%$ of California mental health treatment facilities offered peer services versus $24.6 \%$ of US sites. ${ }^{48}$ Less is known 
about the role of PS in delivering sexual health, STI, and HIV services within mental HS settings serving SGMY.

It is under this backdrop of an evolving mental health and HSs delivery landscape for SGMY that we undertook this study. Using the Gelberg-Andersen Model of Behavioral Health Services Utilization, ${ }^{34,35}$ we focus on enabling factors-that is, we describe the extent to which sexual health and HIV services are available to SGMY within publicly funded mental health programs that serve young adults of ages 16-24 years in California's two largest counties: Los Angeles (2019 population: $\sim 10.3$ million) and San Diego counties ( $\sim 3.4$ million). ${ }^{65}$ We also describe the role of peers in delivering these services.

\section{Methods}

Participants

The Los Angeles County Department of Mental Health and San Diego County Department of Behavioral Health Services provided the researchers with lists of programs that served youth of ages 16-24 years who met mental health eligibility criteria (per each program's assessment) and who received outpatient mental health from July 1, 2015, through June 30, 2018. Each program was contacted by phone to describe the study and identify the appropriate respondent. We requested that questionnaire respondents hold a leadership position and be familiar with the program, including the array of services provided and the use of peer specialists. Respondents were primarily program managers and no incentives for participation were provided.

This study was approved by the Human Subjects Research Protections Program at the University of California, San Diego, Los Angeles Department of Mental Health, and San Diego County Department of Behavioral Health Services in accordance with the Privacy Rule of the Health Insurance Portability and Accountability Act of 1996. No client-level data were analyzed for this study.

\section{Data collection and questionnaires}

We implemented computer-assisted self-administered surveys using Qualtrics, ${ }^{\mathrm{TM}}$ a Health Insurance Portability and Accountability Act of 1996 (HIPAA)-compliant cloud-based survey software (Provo, UT) to collect program-level data. The survey was conducted from August 1, 2018, to February 30, 2019. Electronic informed consent was provided by participants before responding to the questionnaires. Individuals received personalized survey links and nonresponders or persons with incomplete surveys received weekly e-mailed reminders. Respondents were offered the option of completing an interviewer-administered survey on a day/time of their choosing.

Respondents reported on program characteristics including (1) the provision of HIV and sexual HSs (described hereunder), (2) SGMY communities targeted by the program for outreach and engagement, and (3) whether the program employed peer specialists. Specifically, participants reported on whether the program provided the following services: HIV education, HIV testing, HIV pre-exposure prophylaxis (PrEP), HIV postexposure prophylaxis, or HIV medications. Participants reported on whether the program provided sexual/reproductive health education, sexual/reproductive health education for SGMY, testing for STIs, treatment for STIs, or condoms. Respondents also indicated which staff member was responsible for delivering the aforementioned services, which were reported on individually.

\section{Statistical methods}

We describe program characteristics including the SGMY subgroups targeted for service delivery and the employment of peer specialists, the provision of specific HIV, STI and sexual HSs, and the use of peer specialists to provide these services. We use Pearson chi-square tests to assess the relationship between program characteristics and the provision of HIV and sexual HSs.

\section{Results}

Table 1 gives the characteristics of 183 programs that provide outpatient mental HSs to youth with SMI in Los Angeles and San Diego counties. We requested surveys from 260 programs, resulting in a response rate of $70 \%$. Overall, $46 \%$ of outpatient programs serving youth in both counties provide HIV or sexual HSs. Forty-two percent of programs reported having a peer specialist on staff and these programs were more likely to provide HIV or sexual HSs $(p=0.009)$.

Programs targeted multiple SGMY communities including lesbian, gay, bisexual, queer, intersex (LGBQI) youth (17\%), transgender youth (14\%), youth who are homeless or at risk of becoming homeless (24\%), youth who are victims of sex trafficking (13\%), youth who are victims of sexual abuse or violence (19\%), youth with substance use disorders (18\%), youth with dual diagnosis (26\%), youth in or exiting the foster care system 
Table 1. Availability of Peer Specialists on Staff and Youth Communities Targeted for Service Delivery by Outpatient Mental Health Programs in Los Angeles and San Diego Counties Stratified by Sexual Health, Sexually Transmitted Infection, and HIV Services Availability

\begin{tabular}{|c|c|c|c|c|c|}
\hline & \multicolumn{2}{|c|}{ All programs } & \multicolumn{2}{|c|}{$\begin{array}{c}\text { Programs targeting a specific youth } \\
\text { community that provide HIV, STI, } \\
\text { or sexual health services }\end{array}$} & \multirow[b]{2}{*}{$p^{a}$} \\
\hline & $N$ & $\%$ & $N$ & $\%$ & \\
\hline Overall & 183 & & 85 & 46 & \\
\hline \multicolumn{6}{|l|}{ Youth communities targeted by program for outreach and engagement } \\
\hline LGBQI youth & 32 & 17 & 20 & 63 & 0.045 \\
\hline Transgender youth & 26 & 14 & 16 & 62 & 0.096 \\
\hline $\begin{array}{l}\text { MSM youth (i.e., male youth who have sex with men, whether } \\
\text { or not they identify as gay or bisexual) }\end{array}$ & 12 & 7 & 7 & 58 & 0.393 \\
\hline Youth who are homeless or at risk of becoming homeless & 44 & 24 & 29 & 66 & 0.003 \\
\hline Youth who are victims of sex trafficking & 24 & 13 & 17 & 71 & 0.010 \\
\hline Youth who are victims of sexual abuse/violence & 35 & 19 & 21 & 60 & 0.074 \\
\hline Youth with substance use disorders & 33 & 18 & 19 & 58 & 0.157 \\
\hline Youth with dual diagnoses (i.e., mental illness and substance use disorder) & 48 & 26 & 29 & 60 & 0.024 \\
\hline Youth in or exiting the foster care system & 37 & 20 & 23 & 62 & 0.032 \\
\hline Youth involved in the criminal justice system & 39 & 21 & 22 & 56 & 0.160 \\
\hline Parenting or pregnant youth & 21 & 11 & 15 & 71 & 0.015 \\
\hline Peer specialist on staff & 76 & 42 & 44 & 58 & 0.009 \\
\hline
\end{tabular}

Youth are defined as ages $16-24$ years.

${ }^{a}$ For difference in providing HIV or sexual health services among programs targeting a specific youth community.

LGBQI, lesbian, gay, bisexual, queer, intersex; STI, sexually transmitted infection.

(20\%), youth involved in the criminal justice system (20\%), and pregnant or parenting youth (11\%).

Programs that were more likely to provide HIV or sexual HSs were more likely to specifically target LGBQI youth, youth who are homeless or at risk of becoming homeless, youth who are victims of sex trafficking, youth with dual diagnosis, youth in or exiting the foster care system, and pregnant or parenting youth $(p<0.05$ each).

Table 2 gives the types of services available among programs that provide HIV and sexual HSs to youth. Among programs providing services, $64 \%$ provide at least one of the HIV services queried about and $96 \%$ provide at least one type of sexual HS. The most

Table 2. Types of HIV, Sexually Transmitted Infection, and Sexual Health Services Available to Youth in Outpatient Mental Health Programs in Los Angeles and San Diego Counties, Among Programs That Provide at Least One Type of HIV/Sexual Health Service

\begin{tabular}{|c|c|c|c|c|c|c|}
\hline & \multicolumn{2}{|c|}{$\begin{array}{c}\text { Programs providing } \\
\text { HIV/sexual } \\
\text { health services }\end{array}$} & \multicolumn{2}{|c|}{$\begin{array}{l}\text { Programs with peer } \\
\text { specialists who provide } \\
\text { HIV/sexual health } \\
\text { services }\end{array}$} & \multicolumn{2}{|c|}{$\begin{array}{l}\text { Programs with peer } \\
\text { specialists where peers } \\
\text { provide HIV/sexual } \\
\text { health services }\end{array}$} \\
\hline & $N$ & $\%$ & $N$ & $\%$ & $N$ & $\%$ \\
\hline Overall & 85 & & 44 & 52 & & \\
\hline Provides HIV services & 55 & 64 & & & & \\
\hline HIV education & 53 & 62 & 27 & 51 & 11 & 41 \\
\hline HIV testing & 5 & 6 & 3 & 60 & 2 & 67 \\
\hline HIV PrEP & 5 & 6 & 5 & 100 & 1 & 20 \\
\hline HIV PEP & 3 & 4 & 3 & 100 & 1 & 33 \\
\hline HIV medications (e.g., refills) & 6 & 7 & 4 & 67 & 0 & 0 \\
\hline Provides STI and sexual health services & 81 & 95 & & & & \\
\hline Sexual/reproductive health education & 69 & 81 & 34 & 49 & 15 & 44 \\
\hline Sexual/reproductive health education for SGMY & 59 & 69 & 32 & 54 & 14 & 44 \\
\hline STI testing & 5 & 6 & 4 & 80 & 1 & 25 \\
\hline STI treatment & 4 & 5 & 4 & 100 & 0 & 0 \\
\hline Condom distribution & 38 & 45 & 26 & 69 & 13 & 50 \\
\hline
\end{tabular}

Youth are defined as ages $16-24$.

PEP, postexposure prophylaxis; PrEP, pre-exposure prophylaxis; SGMY, sexual and gender minority youth. 
common services were HIV education (62\%), sexual/reproductive health education (81\%), sexual/reproductive health education activities tailored for SGMY (69\%), and condom distribution (45\%). Fewer than $10 \%$ of programs provide HIV testing, HIV PrEP, HIV medication refills, or testing or treatment for STIs.

Peers often provided HIV and sexual HSs (Table 2). Overall, 52\% of programs providing HIV and sexual HSs had a peer specialist on staff. Among these programs, $>40 \%$ involve their peer specialists in the provision of HIV education, HIV testing, sexual/reproductive health education, and condom distribution.

\section{Discussion}

This study aimed to address gaps in the research literature describing sexual health, STI, and HIV services offered to SGMY living with mental health challenges and served by publicly funded outpatient mental health programs. We surveyed a large number of programs in San Diego and Los Angeles counties, which are large and racially/ethnically diverse communities in the state. Research studies such as this one are critically needed to help reduce disparities in mental health status as it overlaps with vulnerabilities experienced by subgroups of SGMY. Overall, we found that programs that included peer providers within their staffing models were more likely to provide any sexual health and HIV services. Our study illustrates the challenges that youth living with mental illness may face in accessing sexual health and HIV services through mental health outpatient programs and underscores potential areas of expansion when addressing the integration of mental health and sexual HSs for youth and SGMY living with SMI.

Prior literature asserts that less has been done to identify and meet the needs of SGMY in health care settings. ${ }^{66,67}$ However, youth and SGMY can benefit from accessing tailored services particularly because of the layered experiences of stigma and discrimination that contribute to diverse and complex physical and mental health and social service needs. ${ }^{1-4}$ New research in this area will be required to understand the impact of youth-focused services on clients' outcomes. ${ }^{68,69}$ In $2017,14.8 \%$ of U.S. community mental health centers offered treatment programs of groups specifically dedicated to SGMY clients. ${ }^{48}$ A 2015 survey of publicly funded mental health programs that serve youth throughout California found that SGMY clients constituted an important target population (LGBTQI: 49\%), suggesting that Southern California programs may have benefited from California's Mental Health Services Act to create new services for SGMY subgroups. ${ }^{47}$

Our analyses demonstrated that inclusion of peer providers in the program was associated with greater delivery of sexual health and HIV services. A program's use of peer providers may be a marker of organizational preferences for innovation or a funding structure that also allows the program to provide sexual HSs. Peers providers have been demonstrated to increase clients' likelihood of returning for annual visits and reducing risky sexual behavior among SGMY. ${ }^{59,61}$ Thus, mental health programs that serve SGMY may consider engaging SGMY peer providers within their service teams.

\section{Limitations}

Our study relied on survey data and may be impacted by recall bias or respondents' familiarity with clinic operations and staffing responsibilities. In addition, responses may have been accurate as of the time the survey was completed, though conditions may have changed since data collection was terminated. Nevertheless, the large sample of participating programs provides a snapshot of services available to SGMY.

\section{Conclusion}

Given SGMY's life stage, mental health programs that target youth and SGMY communities should consider ensuring that sexual HSs are included within their service array to address health disparities experienced by youth living with mental health challenges and SGMY. A one-stop-shop approach may be effective in addressing multiple challenges to SGMY's service utilization as identified by the Gelberg-Andersen Behavioral Model of Health Services Utilization for Vulnerable Populations. ${ }^{39,40}$ Moreover, an integrated system of care may reduce logistical, systemic, trust, and economic barriers to sexual health and HIV service needs that contribute to disparities in these areas among vulnerable youth and SGMY. Adopting peer providers may aid in the implementation of these services by reducing stigma around service utilization and promoting trust among clients and potential clients. Additional research may better elucidate the barriers to the provision of HIV and sexual HSs and the use of peers within programs that serve SGMY clients.

Future studies may consider including other California counties and states to better assess whether the delivery of sexual health and HIV services is prevalent as well as barriers and facilitators to including these services within mental health programming. In addition, 
cost-effectiveness studies can demonstrate the impact of these services on SGMY clients' mental and physical health outcomes and racial/ethnic disparities in HIV and STI rates.

\section{Acknowledgments}

We are grateful to the participating clinics for sharing their time with us; without them, this study would not have been possible. This study was approved by the Human Subjects Research Protections Program at the University of California, San Diego, Los Angeles Department of Mental Health, and San Diego County Department of Behavioral Health Services in accordance with the Privacy Rule of the Health Insurance Portability and Accountability Act of 1996.

\section{Author Disclosure Statement}

No competing financial interests exist.

\section{Funding Information}

We recognize funding from the National Institute of Health, National Institute on Minority Health and Health Disparities: Grant Nos.: 5R01MD011528-02 and 3R01MD011528-02S1.

\section{References}

1. Ghavami N, Katsiaficas D, Rogers LO. Chapter 2: Toward an intersectional approach in developmental science: The role of race, gender, sexual orientation, and immigrant status. In: Advances in Child Development and Behavior. Vol. 50. Horn SH, Ruck MD, Liben LS (Eds). Cambridge, MA: Elsevier, 2016, pp. 31-73.

2. Hughes DL, Watford JA, Del Toro J. A transactional/ecological perspective on ethnic-racial identity, socialization, and discrimination. In: Advances in Child Development and Behavior. Vol. 51. Horn SH, Ruck MD, Liben LS (Eds). Cambridge, MA: Elsevier, 2016, pp. 1-41.

3. Nadal KL, Issa M-A, Leon J, et al. Sexual orientation microaggressions: "Death by a thousand cuts" for lesbian, gay, and bisexual youth. J LGBT Youth. 2011:8:234-259.

4. Russell ST, Fish JN. Mental health in lesbian, gay, bisexual, and transgender (LGBT) youth. Annu Rev Clin Psychol. 2016;12:465-487.

5. Poteat VP, Scheer JR, Mereish EH. Factors affecting academic achievement among sexual minority and gender-variant youth. In: Advances in Child Development and Behavior. Vol. 47. Liben LS, Bigler RS (Eds). San Diego, CA: Elsevier, 2014, pp. 261-300.

6. Division of Adolescent and School Health. Youth Risk Behavior Survey Data Summary \& Trends Report 2007-2017. Atlanta, GA: Centers for Disease Control and Prevention, 2018.

7. Lee YM, Cintron A, Kocher S. Factors related to risky sexual behaviors and effective STI/HIV and pregnancy intervention programs for African American adolescents. Public Health Nurs. 2014:31:414-427.

8. Lee YM, Dancy B, Florez E, Holm K. Factors related to sexual practices and successful sexually transmitted infection/HIV intervention programs for Latino adolescents. Public Health Nurs. 2013;30:390-401.

9. Balaji $A B, A n Q$, Smith $J C$, et al. High human immunodeficiency virus incidence and prevalence and associated factors among adolescent sexual minority Males-3 Cities, 2015. Clin Infect Dis. 2018;66:936-944.

10. Meyer IH. Prejudice, social stress, and mental health in lesbian, gay, and bisexual populations: conceptual issues and research evidence. Psychol Bull. 2003;129:674-697.

11. Herek GM, Gillis JR, Cogan JC. Psychological sequelae of hate-crime victimization among lesbian, gay, and bisexual adults. J Consult Clin Psychol. 1999;67:945-951.
12. Almeida J, Johnson RM, Corliss HL, et al. Emotional distress among LGBT youth: the influence of perceived discrimination based on sexual orientation. J Youth Adolesc. 2009;38:1001-1014.

13. Schnurr PP, Green BL. Understanding relationships among trauma, posttramatic stress disorder, and health outcomes. Adv Mind Body Med. 2004; 20:18-29.

14. Stone DM, Luo F, Ouyang L, et al. Sexual orientation and suicide ideation, plans, attempts, and medically serious attempts: evidence from local Youth Risk Behavior Surveys, 2001-2009. Am J Public Health. 2014;104: 262-271.

15. Friedman MS, Marshal MP, Guadamuz TE, et al. A meta-analysis of disparities in childhood sexual abuse, parental physical abuse, and peer victimization among sexual minority and sexual nonminority individuals. Am J Public Health. 2011;101:1481-1494.

16. Pakula B, Shoveller J, Ratner PA, Carpiano R. Prevalence and co-occurrence of heavy drinking and anxiety and mood disorders among gay, lesbian, bisexual, and heterosexual Canadians. Am J Public Health. 2016:106:1042-1048.

17. Haas AP, Eliason M, Mays VM, et al. Suicide and suicide risk in lesbian, gay, bisexual, and transgender populations: review and recommendations. J Homosex. 2011;58:10-51.

18. Saewyc E, Skay C, Richens K, et al. Sexual orientation, sexual abuse, and HIV-risk behaviors among adolescents in the Pacific Northwest. Am J Public Health. 2006;96:1104-1110.

19. Ybarra ML, Rosario M, Saewyc E, Goodenow C. Sexual behaviors and partner characteristics by sexual identity among adolescent girls. J Adolesc Health. 2016:58:310-316.

20. Kann L, Olsen EO, McManus T, et al. Sexual identity, sex of sexual contacts, and health-risk behaviors among students in grades 9-12-youth risk behavior surveillance, selected sites, United States, 2001-2009. MMWR Surveill Summ. 2011;60:1-133.

21. Tyler KA, Schmitz RM. A comparison of risk factors for various forms of trauma in the lives of lesbian, gay, bisexual and heterosexual homeless youth. J Trauma Disassoc. 2018;19:431-443.

22. McLaughlin KA, Hatzenbuehler ML, Xuan Z, Conron KJ. Disproportionate exposure to early-life adversity and sexual orientation disparities in psychiatric morbidity. Child Abuse Negl. 2012;36:645-655.

23. Marshal MP, Friedman MS, Stall R, et al. Sexual orientation and adolescent substance use: a meta-analysis and methodological review. Addiction. 2008;103:546-556.

24. Talley AE, Hughes TL, Aranda F, et al. Exploring alcohol-use behaviors among heterosexual and sexual minority adolescents: intersections with sex, age, and race/ethnicity. Am J Public Health. 2014;104:295-303.

25. Cutuli J, Treglia D, Herbers JE. Adolescent homelessness and associated features: prevalence and risk across eight states. Child Psychiatry Hum Dev. 2020;51:48-58.

26. Choi SK, Wilson BDM, Shelton J, Gates G. Serving Our Youth 2015: The Needs and Experiences of Lesbian, Gay, Bisexual, Transgender, and Questioning Youth Experiencing Homelessness. Los Angeles: The Williams Institute with True Colors Fund, 2015.

27. Wayman RH, LaKesha P. Incidence and Vulnerability of LGBTQ Homeless Youth. Washington, DC, USA: National Alliance to End Homelessness, 2008.

28. Graham R, Berkowitz B, Blum R, et al. The Health of Lesbian, Gay, Bisexual, and Transgender People. Washington, DC: National Academies Press (US), 2011.

29. Chan CD, Henesy RK. Navigating intersectional approaches, methods, and interdisciplinarity to health equity in LGBTQ + communities. J LGBT Issues Counsel. 2018;12:230-247.

30. Clatts MC, Goldsamt L, Yi H, Gwadz MV. Homelessness and drug abuse among young men who have sex with men in New York city: a preliminary epidemiological trajectory. J Adolesc Health. 2005;28:201-214.

31. Morton MH, Dworsky A, Matjasko JL, et al. Prevalence and correlates of youth homelessness in the United States. J Adolesc Health. 2018;62: 14-21.

32. Whitbeck LB, Chen X, Hoyt DR, et al. Mental disorder, subsistence strategies, and victimization among gay, lesbian, and bisexual homeless and runaway adolescents. J Sex Res. 2004;41:329-342.

33. Johnson TP, Graf I. Unaccompanied Homeless Youth in Illinois: 2005. Chicago, IL: Survey Research Laboratory College of Urban Planning and Public Affairs University of Illinois at Chicago, 2005. 
34. Gelberg L, Andersen RM, Leake BD. The Behavioral Model for Vulnerable Populations: application to medical care use and outcomes for homeless people. Health Serv Res. 2000;34:1273.

35. Stein JA, Andersen R, Gelberg L. Applying the Gelberg-Andersen behavioral model for vulnerable populations to health services utilization in homeless women. J Health Psychol. 2007;12:791-804.

36. Andersen R. National health surveys and the behavioral model of health services use. Med Care. 2019;46:647-653.

37. Stein JA, Andersen RM, Robertson M, Gelberg L. Impact of hepatitis B and $C$ infection on health services utilization in homeless adults: a Test of the Gelberg-Andersen Behavioral Model for Vulnerable Populations. Health Psychol. 2012;31:20-30.

38. Small LFF. Use of mental health services among people with co-occurring disorders and other mental health co-morbidities: employing the behavioral model of vulnerable populations. Ment Health Subst Use. 2010;3:81-93.

39. Mayer $\mathrm{KH}$, Bradford JB, Makadon $\mathrm{HJ}$, et al. Sexual and gender minority health: what we know and what needs to be done. Am J Public Health. 2011;98:989-995.

40. Tanner AE, Philbin MM, Chambers BD, et al. Healthcare transition for youth living with HIV: outcomes from a prospective multi-site study. J Adolesc Health. 2018;63:157-165.

41. UCLA Center for Health Policy Research. Current insurance status of transition age youth (California). California Health Interview Survey (CHIS) Database. 2017. Available at http://ask.chis.ucla.edu Accessed September 10, 2019.

42. Albuquerque GA, de Lima Garcia C, da Silva Quirino G, et al. Access to health services by lesbian, gay, bisexual, and transgender persons: systematic literature review. BMC Int Health Hum Rights. 2016;16:2.

43. Watts J, O'Byrne P. 'I don't care if you think I'm gay... that won't make me either promiscuous or HIV positive': HIV, stigma, and the paradox of the gay men's sexual health clinic - an exploratory study. Appl Nurs Res. 2019;47:1-3.

44. Jahn JL, Bishop RA, Tan ASL, Agenor M. Patient-provider sexually transmitted infection prevention communication among young adult sexual minority cisgender women and nonbinary assigned female at birth individuals. Womens Health Issues. 2019;29:308-314.

45. Fisher $C B$, Fried AL, Desmond M, et al. Perceived barriers to HIV Prevention Services for transgender youth. LGBT Health. 2018;5:350-358.

46. Clark W, Welch SN, Berry SH, et al. California's historic effort to reduce the stigma of mental illness: the Mental Health Services Act. Am J Public Health. 2013;103:786-794.

47. Ojeda VD, Hiller SP, Hurst $S$, et al. Implementation of age-specific services for transition-age youths in California. Psychiatr Serv. 2016;67:970-976.

48. SAMHSA. National Mental Health Services Survey (N-MHSS): 2017, Data On Mental Health Treatment Facilities. 2017. Available at https://www .samhsa.gov/data/sites/default/files/cbhsq-reports/2017_National_ Mental_Health_Services_Survey.pdf Accessed September 10, 2019.

49. Chuah FLH, Haldane VE, Cervero-Liceras F, et al. Interventions and approaches to integrating HIV and mental health services: a systematic review. Health Policy Plann. 2019;32(suppl_4):iv27-iv47.

50. Adams J, Pollard RS, Sikkema KJ. Feasibility of integrated depression care in an HIV Clinic. Psychiatr Serv. 2011;62:804.

51. Williams KA, Chapman MV. Comparing health and mental health needs, service use, and barriers to services among sexual minority youths and their peers. Health Soc Work. 2011;36:197-206.

52. Patel VV, Ginsburg Z, Golub SA, et al. Empowering with PrEP (E-PrEP), a peer-led social media-based intervention to facilitate HIV preexposure prophylaxis adoption among young Black and Latinx Gay and Bisexual Men: protocol for a Cluster Randomized Controlled Trial. JMIR Res Protoc. 2018;7:e11375

53. Golden MR, Gift TL, Brewer DD, et al. Peer referral for HIV case-finding among men who have sex with men. AIDS. 2006;20:1961-1968.

54. Shangani S, Escudero D, Kirwa K, et al. Effectiveness of peer-led interventions to increase HIV testing among men who have sex with men: a systematic review and meta-analysis. AIDS Care. 2017;29:1003-1013.

55. Taegtmeyer M, Hightower A, Opiyo W, et al. A peer-led HIV counselling and testing programme for the deaf in Kenya. Disabil Rehabil. 2009;31: 508-514.
56. Denno DM, Hoopes AJ, Chandra-Mouli V. Effective strategies to provide adolescent sexual and reproductive health services and to increase demand and community support. J Adolesc Health. 2015;56(1 Suppl): S22-S41.

57. Davis TS, Saltzburg S, Locke CR. Supporting the emotional and psychological well being of sexual minority youth: youth ideas for action. Child Youth Serv Rev. 2009;31:1030-1041.

58. Wells EA, Asakura K, Hoppe MJ, et al. Social services for sexual minority youth: preferences for what, where, and how services are delivered. Child Youth Serv Rev. 2012;36:312-320.

59. Brindis CD, Geierstanger SP, Wilcox N, et al. Evaluation of a peer provider reproductive health service model for adolescents. Perspect Sex Reprod Health. 2005;37:85-91.

60. Lloyd-Evans B, Mayo-Wilson E, Harrison B, et al. A systematic review and meta-analysis of randomised controlled trials of peer support for people with severe mental illness. BMC Psychiatry. 2014;14:39.

61. Reif S, Braude L, Lyman DR, et al. Peer recovery support for individuals with substance use disorders: assessing the evidence. Psychiatr Serv. 2014;65:853-861.

62. Solomon P. Peer support/peer provided services underlying processes, benefits, and critical ingredients. Psychiatr Rehabil J. 2004;27: 392-401.

63. Alegria $M$, Chatterji $P$, Wells $K$, et al. Disparity in depression treatment among racial and ethnic minority populations in the United States. Psychiatr Serv. 2008;59:1264-1272.

64. Bonnie R, Stroud C, Breiner H. Investing in the Health and WellBeing of Young Adults. Washington, DC: National Academies Press (US), 2015.

65. State of California Department of Finance. E-1 Population Estimates for Cities, Counties and the State with Annual Percent Change-January 1, 2018 and 2019. 2019. Available at www.dof.ca.gov/Forecasting/ Demographics/Estimates/e-1 Accessed November 1, 2019.

66. Mattocks KM, Kauth MR, Sandfort T, et al. Understanding health-care needs of sexual and gender minority veterans: how targeted research and policy can improve health. LGBT Health. 2013;1:50-57.

67. Neville $S$, Henrickson M. Perceptions of lesbian, gay and bisexual people of primary healthcare services. J Adv Nurs. 2006;55:407-415.

68. Abbott J-AM, Klein B, McLaren S, et al. Out \& online: study protocol for a randomised controlled trial investigating the effectiveness of a tailored online multi-symptom mental health and wellbeing program for samesex attracted young adults. Trials. 2014;15:504-513.

69. Pepping CA, Lyons A, McNair R, et al. A tailored compassion-focused therapy program for sexual minority young adults with depressive symotomatology: study protocol for a randomized controlled trial. BMC Psychol. 2017;5:1-7.

Cite this article as: Clermont $\mathrm{D}$, Gilmer T, Burgos $\mathrm{J}$, Berliant $\mathrm{E}$ Ojeda VD (2020) HIV and sexual health services available to sexual and gender minority youth seeking care at outpatient public mental health programs in two California counties, Health Equity 4:1, 375-381, DOI: 10.1089/heq.2020.0014.

\section{Abbreviations Used}

$\mathrm{HS}=$ health service

$\mathrm{LGBQI}=$ lesbian, gay, bisexual, queer, intersex

$\mathrm{PEP}=$ postexposure prophylaxis

PrEP $=$ pre-exposure prophylaxis

$\mathrm{PS}=$ peer support

$\mathrm{SGM}=$ sexual and gender minority

$S G M Y=$ sexual and gender minority youth

$\mathrm{SMI}=$ serious mental illness

$\mathrm{STI}=$ sexually transmitted infection 